\title{
Atraumatic Restorative Treatment in the UK
}

\author{
UK dentists' attitudes and behaviour towards Atraumatic Restorative Treatment for primary teeth \\ F. J. T Burke, S. McHugh, L Shaw, M-T. Hosey, L. Macpherson, S. Delargy and B. Dopheide Br Dent J 2005; 199: \\ 365-369
}

\section{Background}

Atraumatic Restorative Treatment (ART) was introduced a decade ago as a minimal intervention treatment for caries in unindustrialised countries, but UK general dental practitioners (GDPs) may also be using this technique.

Objective

This study aimed to determine the materials and techniques used by a group of UK GDPs to treat caries in primary teeth.

Method

A questionnaire, designed to determine GDPs' use of materials and techniques in the restoration of caries in primary teeth, was distributed to 600 GDPs in Scotland and England, with an explanatory letter and reply-paid envelope. The questionnaire included colour illustrations of two carious cavities in primary molar teeth and a request that respondents draw the cavity outline that they would use on the illustrations. The cavity outlines were assessed independently by two examiners. All other data were collated and analysed.

Results

390 usable replies were received, a response rate of 65\%. Of the respondents, 99\% treated child patients and 42\% of respondents were aware of ART. For treatment of a small Class II cavity, 37\% drew a cavity outline without extension beyond removal of caries and a majority suggested use of an adhesive material (51\% glass ionomer, 13\% compomer). For cavity preparation, 47\% of respondents used a drill, 10\% an excavator and 41\% used both. For treatment of a large occluso-lingual cavity, again most used an adhesive technique (44\% glass ionomer, 12\% compomer) for its restoration, while 50\% used a drill, 7\% an excavator and 42\% used both for cavity preparation.

Conclusion

Most respondents used adhesive materials for restoration of caries in primary molars, but, despite $42 \%$ of respondents stating that they were aware of the treatment, 'true' ART was adopted by fewer than $10 \%$ of respondents.

\section{N BRIEF}

- Almost half of the practitioners surveyed were aware of the Atraumatic Restorative Treatment technique (ART).

- Few practitioners, however, followed ART principles in their practice.

- Further education on minimal intervention techniques would seem desirable.

\section{COMMENT}

The oral health care service is innocent of failing to prevent dental caries in children; the obloquy for that rests on society as a whole. ${ }^{1}$ In this lamentable state of affairs general dental, community and academic services strive to provide ever more kindly and effective care. This study of atraumatic restorative treatment (ART) for children with decayed deciduous teeth explores how well the technique is being used. Trauma may be emotional as well as physical and affect providers of care ${ }^{2}$ as well as young patients; if it can be avoided so much the better. To varying degrees local anaesthesia and the drill are traumatic: ART posits that they may be unnecessary.

Findings about five-year-olds' deciduous teeth reveal that levels of decay over the last two years are unchanged and one in 25 children in 16 PCTs have a dental abscess or draining sinus. ${ }^{3}$ Levine et $a l_{.}{ }^{4}$ found that, using pain or sepsis as an outcome, no difference existed between restored and unrestored teeth; of the unrestored only (sic) $16 \%$ went on to cause pain requiring treatment or extraction. Studies of dental pain and how it affects children's quality of life ${ }^{5}$ would appear to support BSPD's ${ }^{6}$ clinical guidelines stating that the primary dentition should be restored.

Although $42 \%$ of 390 respondents knew of ART, (and presumably its tenets), few practised it. Moreover, of those who did, less than $10 \%$ adopted the 'true' ART approach and thus the proportion in the UK must be negligible.

As it stands ART might well be viewed as a way of legitimising inferior care using rudimentary instruments and crude techniques. If methods of treating carious deciduous teeth continue in disarray, as at present, we must be prepared for it to foster litigation. A 'fat face' in a regularly attending five-year-old conjures up a pattern of care only supportable forensically if BSPD guidelines have been followed, and even then with difficulty.

ART calls for more research and a recent study in seven-year-olds 7 is supportive because it suggests that partial removal of caries may not prejudice the durability and effectiveness of a restoration. If, in due course, research confirms ART's merits it must then, surely, develop an academically derived protocol for its practice and for which there is consensus. It can then feature in undergraduate and postgraduate teaching and be promoted universally.

D. W. Sarll, Past president, British Association for the Study of Community Dentistry doi: 10.1038/sj.bdj.4812697

1. Sarll D W. Who is responsible for good oral health? Br Dent J 1996; 180: 164-167.

2. Pine C. etal. Barriers to the treatment of childhood caries perceived by dentists working in different countries. Comm Dent Health 2004; 21: 112-120.

3. Pitts N B, Boyles J, Nugent Z J, Thomas N, Pine CM. The dental caries experience of 5year-old children in England and Wales (2003/4) and in Scotland (2002/3). Comm Dent Health 2005: 22: 46-56.

4. Levine R S, Pitts N B, Nugent N J. What happens to unrestored carious deciduous teeth? BrDent J 2002; 193: 99-103.

5. Slade G D. Epidemiology of dental pain and dental caries among children and adolescents. Comm Dent Health 2001; 18: 219-227.

6. British Society of Paediatric Dentistry. A policy document on management of caries in the primary dentition. Int J Paed 2001; 11: 153-157.

7. Foley J, Evans D, Blackwell A. Partial caries removal and cariostatic materials. Br DentJ 2004; 196: 697-701. 